\title{
Can magnetic breakout be achieved from multiple flux emergence?
}

\author{
D. MacTaggart and A. W. Hood
}

\author{
School of Mathematics and Statistics, University of St. Andrews, North Haugh, St. Andrews, Fife, KY16 9SS, UK \\ e-mail: dm428@st-andrews.ac.uk
}

Received 28 January 2009 / Accepted 5 March 2009

\begin{abstract}
Aims. We study the breakout model using multiple flux emergence to produce the magnetic configuration and the trigger. We do not impose any artificial motions on the boundaries. Once the original flux tube configuration is chosen the system is left to evolve itself. Methods. We perform non-linear simulations in 2.5D by solving the compressible and resistive MHD equations using a Lagrangian remap, shock capturing code (Lare2D). To produce a quadrupolar configuration from flux emergence we build on previous work where the interaction of two flux tubes forms the required quadrupole. Instead of imposing a shearing flow, a third flux tube is then allowed to emerge up through the central arcade.

Results. Breakout is not achieved in any of the experiments. This is due to the interaction of the third tube with the quadrupole and the effect of the plasma $\beta$ being $O(1)$ at the photosphere and $\beta \gtrsim O(1)$ in the solar interior. When $\beta$ is of these orders, flows generated in the plasma can influence the magnetic field and so photospheric footpoints do not remain fixed.
\end{abstract}

Key words. Sun: magnetic fields - magnetohydrodynamics (MHD) - methods: numerical

\section{Introduction}

The challenge of modelling and predicting coronal mass ejections (CMEs) has been one of the main objectives of solar theorists over the past few decades. Many different types of models have been suggested for the triggering and evolution of such phenomena. These include a mixture of ideal (Török \& Kliem 2003) or nonideal MHD (Antiochos et al. 1999; Linker et al. 2003) and force-free (Mackay \& van Ballegooijen 2006a,b; Yeates \& Mackay 2009a) or non-force-free magnetic fields (Forbes 2000). Yeates \& Mackay (2009b) study the global evolution of CMEs with a magnetofrictional model that uses Kitt Peak synoptic magnetograms as input. Here we shall focus on the nonideal subset as being relevant to our study.

Nonideal models (or resistive MHD models) appeal to processes such as reconnection to bypass the Aly-Sturrock constraint (Aly 1991; Sturrock 1991) which severely restricts ideal models. One such model that has received much attention over recent years is the breakout model (Antiochos et al. 1999). The initial condition for the breakout model is a quadrupolar magnetic field. There are four flux domains. Two consist of flux joining the neighbouring bipole polarity and the inner flux region where the innermost polarities connect. In the outer flux region the magnetic field joins the outer two polarities above the other regions. The initial flux in the inner region must exceed the flux in the outer region for breakout to occur.

To initiate an eruption, this configuration is stressed by shearing the central arcade through an imposed photospheric flow on the lower boundary. The X-point becomes distorted into a horizontal current layer. As the shearing increases, the diffuse current evolves into a thin current sheet that results in rapid reconnection. This reconnection allows for the transfer of magnetic flux from the inner and overlying systems to the side arcades. The loss of the field above the sheared central arcade leads to a runaway expansion. This, together with the increase in magnetic pressure due to the additional flux from reconnection at the the top current sheet, leads to the formation of a current sheet in the central arcade. Reconnection here results in the expulsion of a flux rope. There have been several simulations of breakout in 2.5D (Antiochos et al. 1999; MacNeice et al. 2004; van der Holst et al. 2007) and some recent simulations in 3D (DeVore \& Antiochos 2008; Lynch et al. 2008). Cook et al. (2009) have extrapolated global potential fields from magnetograms to find breakout topologies. A recent 2.5D simulation has been performed by Zuccarello et al. (2008) where, instead of shearing, they drive breakout by flux emergence. Here flux emergence is simulated by imposing a time-dependent condition on the photospheric boundary. In their simulation they produce a CME from a detached helmet streamer and not from the pinching off of a flux rope from the central arcade. Hence, the topology of their eruption is different from that in Antiochos et al. (1999).

Before the breakout model was proposed, there were breakout-like simulations that modelled plasmoid ejections in a 2D cartesian geometry. In these models, interacting arcades are sheared until a flux rope is expelled upwards. Mikic et al. (1988) took a (laterally restricted) periodic arcade setup and applied a shear flow so that the arcades would be energized in a symmetric way. Numerical asymmetries allowed reconnection to occur at the periodic boundaries and a plasmoid formed and erupted. This would obviously not form in an ideal simulation. Biskamp \& Welter (1989) considered a more realistic setup where the arcades are not neccessarily symmetric and not confined by lateral boundary conditions. They found that plasmoid ejection is only possible if the interaction of the arcades is strong and that only a triple of appropriately packed arcades leads to fast plasmoid ejection.

None of the above models have been directly driven by particular observations, rather, the breakout model has been 
designed to bypass the Aly-Sturrock constraint. There have been many observational studies of CMEs with a mixture of results. Some work claims to find breakout-compatible eruptions (Li \& Luhmann 2006) and others find eruptions that are incompatible (Ugarte-Urra et al. 2007). A comprehensive list of observational papers related to the breakout model is given in Lynch et al. (2008). Observational work has also tried to find the link between CME eruption and flux emergence. Zhang et al. (2008) published a statistical survey of $189 \mathrm{CME}$-source regions, 46 active regions and 15 newly emerging active regions. They find that $60 \%$ of the CME-source regions have a flux increase during $12 \mathrm{~h}$ before the eruption and $40 \%$ show a magnetic flux decrease. They conclude that the relationship between CMEs and flux emergence is complicated and that flux emergence alone is not unique as a trigger for a CME.

What all of the breakout models and simulations, mentioned above, have in common is that the magnetic configuration (e.g. quadrupolar) is set up as the initial condition and is dynamically altered by imposed photospheric motions, whether they be shearing motions or changing the field to emulate emergence. In this paper we study the breakout model using a more self-consistent method. We carry out 2.5D MHD simulations of three twisted flux tubes as they rise from the solar interior, through the photosphere and into the corona. The idea is that the first two tubes will create the quadrupolar structure and the third will emerge into the central arcade above the photosphere. This results in an increased magnetic pressure in the central arcade. Hence the magnetic pressure is increased by emergence rather than imposed shearing. This is self-consistent compared with the models previously described because it does not artificially impose any flows on the boundaries. It is also based on multiple flux emergence which is a natural occurence on the Sun. The initial condition is chosen and the system is left to evolve itself. The choice of initial condition is designed to produce the magnetic configuration of the breakout model in the solar atmosphere. Of course, on the Sun, other combinations are possible but here we are studying whether or not breakout can be achieved from multiple flux emergence. We shall now give a brief synopsis of relevant flux emergence simulations.

There have been several simulations that model flux emerging into a pre-existing horizontal magnetic field in the corona (Shibata et al. 1989; Archontis et al. 2005; Galsgaard et al. 2007). Archontis et al. (2007) performed 2.5D simulations of the interaction of two flux tubes. One of the reasons for studying this was in fact to allow one tube to emerge and produce a nonuniform coronal field for the other tube to emerge into. This was achieved by simply placing the two flux tubes at different heights in the solar interior. In the experiment both tubes had the same sign of twist. This means that when the second tube emerges into the expanded field of the other, their magnetic fields point in opposite directions. A curved current sheet forms as the second tube emerges into the field of the first. Between the axes of the two tubes the current sheet is at its strongest. Eventually, the tearing instability takes effect here. The result is a change of topology through the ejection of plasmoids. The central current sheet remains and this relaxes to an X-point.

This produces the initial quadrupolar configuration of the breakout model in a self-consistent fashion. Rather than shear the central arcade, to increase the magnetic pressure, we emerge more flux (i.e. a third flux tube) in the central region. The orientation of the third tube is chosen to retain the quadrupolar nature of the photospheric field.

The paper is organized as follows. Section 2 presents the equations and numerical model used in the experiments.
Section 3 will describe the quadrupolar equilibrium and the effects of introducing a third tube. Finally, Sect. 4 will summarize the conclusions.

\section{The numerical model}

For the numerical experiments we use a $2.5 \mathrm{D}$ version of the Lagrangian remap scheme detailed in Arber et al. (2001). This code was used by Archontis et al. (2007) for modelling the interaction of two flux tubes. All variables are made dimensionless against photospheric values. These values are: pressure, $p_{\mathrm{ph}}=1.4 \times 10^{5} \mathrm{erg} \mathrm{cm}^{-3}$; density, $\rho_{\mathrm{ph}}=3 \times 10^{-7} \mathrm{~g} \mathrm{~cm}^{-3}$; temperature, $T_{\mathrm{ph}}=5.6 \times 10^{3} \mathrm{~K}$ and scale height $H_{\mathrm{ph}}=170 \mathrm{~km}$. The other units used in the simulations are: time, $t_{\mathrm{ph}}=25 \mathrm{~s}$; velocity, $U=\left(p_{\mathrm{ph}} / \rho_{\mathrm{ph}}\right)^{1 / 2}=6.8 \mathrm{~km} \mathrm{~s}^{-1}$ and magnetic field $B_{\mathrm{ph}}=$ $1.3 \times 10^{3} \mathrm{G}$. The evolution of the system is governed by the following time-dependent and resistive (non-dimensionalized) MHD equations.

$$
\frac{\partial \rho}{\partial t}+\nabla \cdot(\rho \boldsymbol{u})=0
$$

$\rho\left(\frac{\partial \boldsymbol{u}}{\partial t}+(\boldsymbol{u} \cdot \nabla) \boldsymbol{u}\right)=-\nabla p+(\nabla \times \boldsymbol{B}) \times \boldsymbol{B}+\rho \boldsymbol{g}$,

$\frac{\partial \boldsymbol{B}}{\partial t}=\nabla \times(\boldsymbol{u} \times \boldsymbol{B})+\eta \nabla^{2} \boldsymbol{B}$

$\rho\left(\frac{\partial \varepsilon}{\partial t}+(\boldsymbol{u} \cdot \nabla) \varepsilon\right)=-p \nabla \cdot \boldsymbol{u}+\eta j^{2}+Q_{\mathrm{visc}}$

with specific energy density

$\varepsilon=\frac{p}{(\gamma-1) \rho}$

The basic variables are the density $\rho$, the pressure $p$, the magnetic field vector $\boldsymbol{B}$ and the velocity vector $\boldsymbol{u} . \boldsymbol{j}$ is the magnitude of current density and $\boldsymbol{g}$ is gravity (uniform in the $y$-direction). $\gamma$ is the ratio of specific heats and is taken as $5 / 3 . \eta$ is the resistivity which is taken to be uniform. We only implement uniform resistivity as it was found by Archontis et al. (2007) that the difference in results between using it and locally enhanced resistivity is slight. For all the experiments we take $\eta=0.001$. The code accurately resolves shocks by using a combination of artificial viscosity and Van Leer flux limiters. In such regions, heating is added to the energy equation and is represented by $Q_{\text {visc }}$.

The initial stratification of the atmosphere is similar to the model used by Archontis et al. (2004). Figure 1 displays the height profiles of temperature, pressure and density. In order to calculate these profiles, the temperature profile is specified and the other variables are found by numerically solving the hydrostatic equation. This gives a numerically stable equilibrium. The solar interior $(y \leq 0)$ is taken as convectively stable since we are only focussing on the emerging field. The photosphere/chromosphere lies in the region $0 \leq y \leq 10$, the transistion region in $10 \leq y \leq 20$ and the corona in $y \geq 20$.

In the initial condition the magnetic flux tubes are placed below the photosphere in the solar interior. For a single tube, the axial magnetic field is given by

$B_{z}=B_{0} \exp \left(-r^{2} / R^{2}\right)$ 


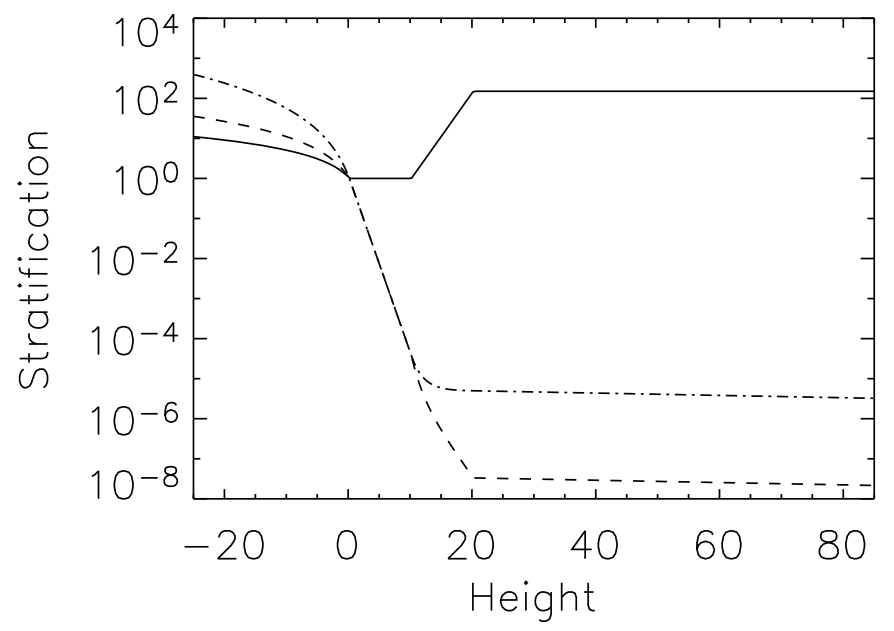

Fig. 1. The initial hydrostatic startification. The curves are temperature (solid), density (dash) and pressure (dot-dash).

where $B_{0}$ is the magnetic field strength on the axis of the tube, $R$ is the radius of the tube and $r=\sqrt{\left(x-x_{0}\right)^{2}+\left(y-y_{0}\right)^{2}}$ is the radial from the axis of the tube, positioned at $\left(x_{0}, y_{0}\right)$. The flux tubes are uniformly twisted and have an azimuthal component given by

$B_{\varphi}=\alpha r B_{z}$.

Here, $\alpha$ is the twist parameter. The sign of this parameter determines if the tube has left or right-handed twist.

To initiate the simulations a density deficit is introduced into the tubes. This technique has become standard practice in flux emergence simulations (Fan 2001; Archontis et al. 2004, 2007; Murray et al. 2006).

One of the important features of this model is that, once the magnetic fields are put into the hydrostatic equilibrium, the size of the plasma beta, $\beta=p /\left(B^{2} / 2 \mu\right)$, varies greatly in the simulation box. In the solar interior, $\beta \gtrsim O(1)$, at the photosphere, $\beta \sim O(1)$ and in the corona, $\beta \sim o(1)$ towards the end of the simulations.

\section{Simulations}

\subsection{Introduction}

As mentioned in Sect. 1 the aim of this paper is to investigate flux rope eruptions, in the framework of the breakout model, by a self-consistent method. This means that the initial configuration (quadrupolar for breakout) should be part of the evolution of the system. Archontis et al. (2007) found, as previously described, that the interaction of two twisted flux tubes can result in the creation of a quadrupolar field. With the formation of this field the next step is to emerge a third flux tube up through the central arcade. The aim is to create the same effect as shearing (increase in $B^{2} / 2 \mu$, rise of X-point, current sheet formation and then reconnection) without actually imposing any artificial shear. Before describing this, however, we shall examine more closely the equilibrium resulting from the emergence of two tubes.

\subsection{Quadrupolar equilibrium}

We perform four numerical experiments, similar to Archontis et al. (2007), to produce a quadrupolar equilibrium from the

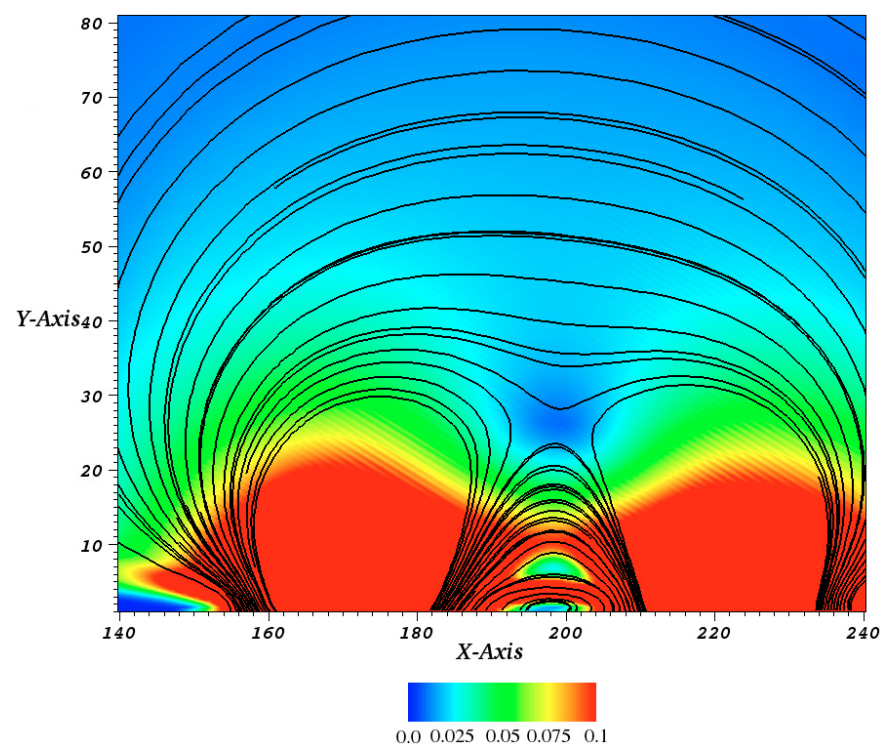

Fig. 2. The resulting quadrupole from experiment (i) where the tubes have an initial lateral separation of 20. The colourmap shows $|\boldsymbol{B}|$ and some fieldlines are traced in black.

emergence and interaction of two flux tubes. We examine the effect of changing the lateral distance between the axes of the initial two tubes. In dimensionless coordinates, the simulation box ranges from 0 to 400 on the $x$-axis and -50 to 140 on the $y$-axis. The side boundaries are periodic and the top and bottom boundaries are closed with damping layers. The tubes are kept at the same strength, twist and radius $\left(B_{0}=5, \alpha=-0.4\right.$, $R=2.5$ ) in all the experiments and only the original positions are changed. The four experiments involve two tubes placed in the solar interior with lateral separation of the axes of (i) 20; (ii) 40; (iii) 60 and (iv) 80 units respectively. The left-hand tube is always placed at $y=-5$, while the right-hand tube is placed at heights $y=-8,-9,-10,-11$, respectively. The reason for this is because when the tubes are further away from each other it takes the left-hand tube longer to expand over the right-hand tube and provide a field for it to emerge into. This means that the righthand tube is slightly weaker, at the same height, for experiments with a larger initial separation. None of the experiments, however, are symmetric and the differences are slight.

The general behaviour of the experiments follows that of Archontis et al. (2007) and is described in Sect. 1. Each case produces a quadrupolar formation. Figure 2 portrays the profile of a typical quadrupolar formation from the experiments. The black contours outline field lines and the colourmap shows the magnitude of the magnetic field. Note that $|\boldsymbol{B}|$ is locally a minimum near the $\mathrm{X}$-point. The $\mathrm{X}$-point is not a null point as there is still an axial component of the magnetic field into the plane of the figure. We will now examine some of the important features of this quadrupolar formation.

\subsubsection{Photospheric conditions}

Here we will consider the field at times long after the formation of the quadrupolar field. These times will vary due to the different initial tube separation of the various cases.

Often in the modelling of solar eruptions the atmosphere is taken to be the corona, with $\beta \sim o(1)$, and the bottom boundary is taken to be the photosphere. Footpoints of a field are assumed to be fixed at this bottom boundary, unless a flow is artificially 


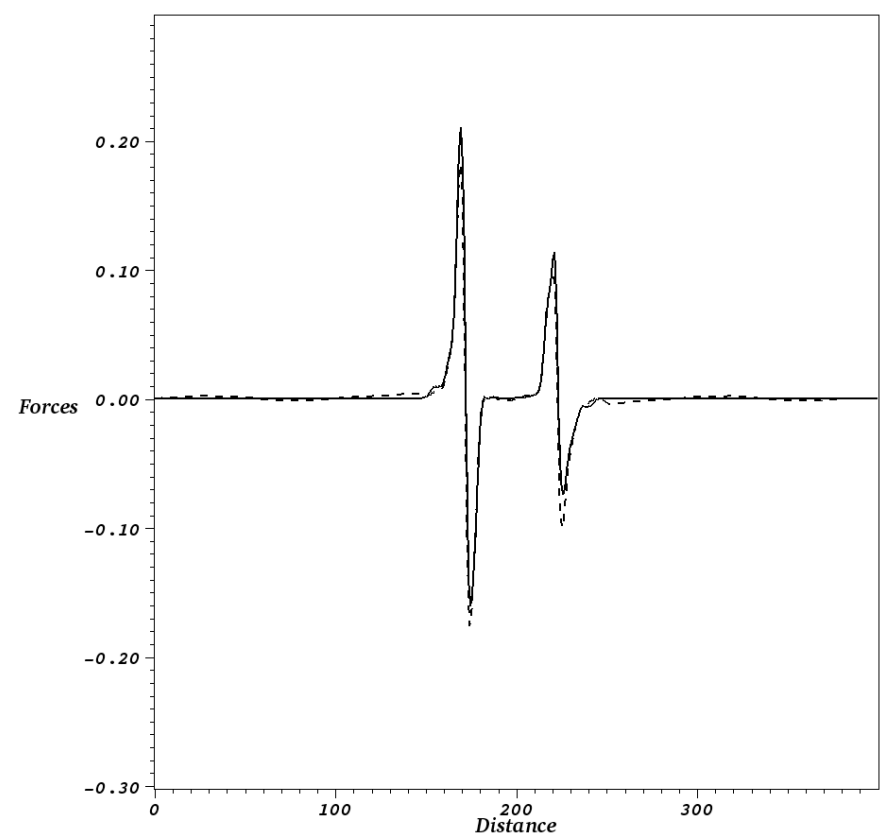

Fig. 3. The solid line shows $(\boldsymbol{j} \times \boldsymbol{B})_{x}$ and the dashed line, $\partial p / \partial x$. Both cuts are taken at the photosphere at time $t=275$ for experiment (i).

imposed. On examination of the forces at the base of the photosphere $(y=0)$ in our experiments, however, the assumption of a fixed field profile at the photosphere is not strictly true. Since, in this region, $\beta \sim O(1)$, flows in the plasma can influence the field and move it. Long after the formation of the quadrupole, the lateral plasma forces approximately balance. For example, the $x$-component of the Lorentz force approximately balances the lateral plasma pressure gradient, $\partial p / \partial x \approx(\boldsymbol{j} \times \boldsymbol{B})_{x}$. This implies there is no horizontal acceleration and that $u_{x}(x, 0, t) \approx$ const. From the simulations, this velocity component is small but non-zero and approximately constant in time. The $z$-component of the Lorentz force gives rise to a shearing motion. However, the magnitude of $u_{z}$ is an order of magnitude smaller than $u_{x}$. Dynamically, the main effect is the lateral spreading of the system. Shearing motion is also obtained in 3D flux emergence simulations (Manchester et al. 2004; Archontis \& Török 2008).

Consider experiment (i) with an initial lateral separation of 20 at time $t=275$. The initial formation of the quadrupolar structure occurs around $t \approx 120$. Figure 3 compares the horizontal component of the Lorentz (solid curve) and plasma pressure gradient (dashed curve) forces at the photospheric base $y=0$. The forces are nearly in balance so that the net force is extremely small. The approximate steady state horizontal flow, at the photospheric base $y=0$, is displayed in Fig. 4. This shows that there remains a steady flow that is slowly moving the magnetic sources apart. The left hand tube is moving to the left and the right hand tube is moving to the right. Although the flows are highly subsonic, it does mean that the magnetic sources do not remain at fixed locations. However, this is exactly what one would expect. If the magnetic field has to be sufficiently strong (i.e. $\beta$ around unity) to emerge, then the Lorentz force and the pressure gradient forces will be large enough to move the photospheric footpoints apart.

If we now consider experiment (iv), with an initial axial separation of 80 , at time $t=338$ (the later time is needed as the quadrupolar structure takes longer to form due to the larger separation of the tubes), the profiles are remarkably similar (compare Figs. 6 with 4 for experiment (i)). It suggests that the initial

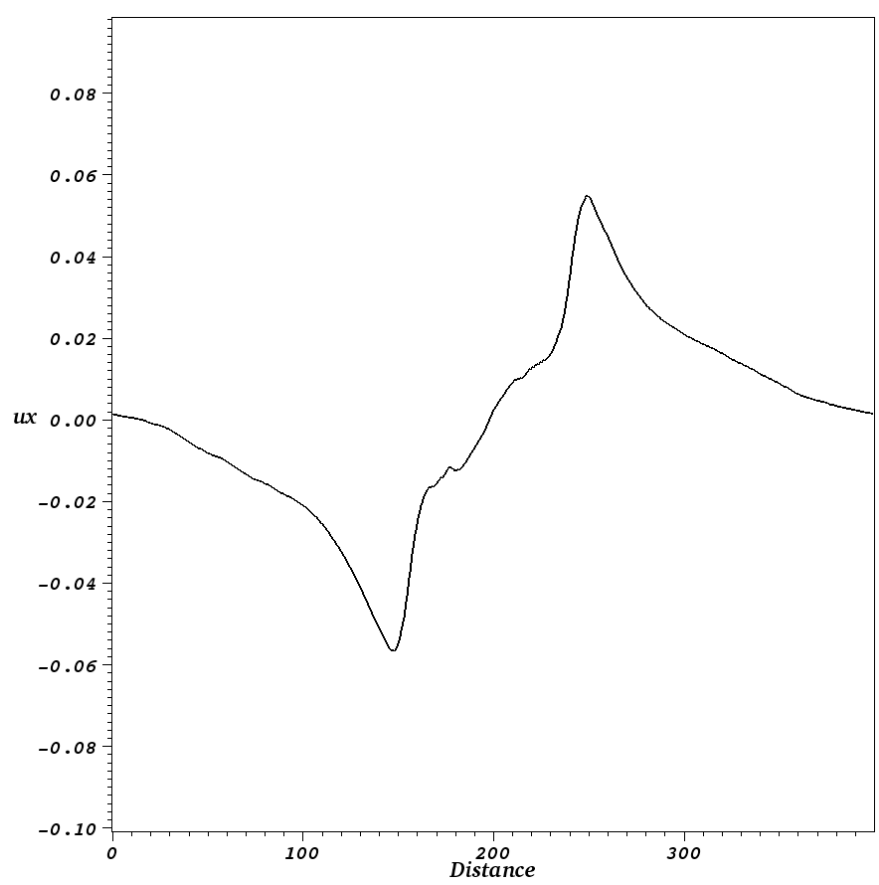

Fig. 4. The horizontal velocity at the photosphere at $t=275$ for experiment (i).

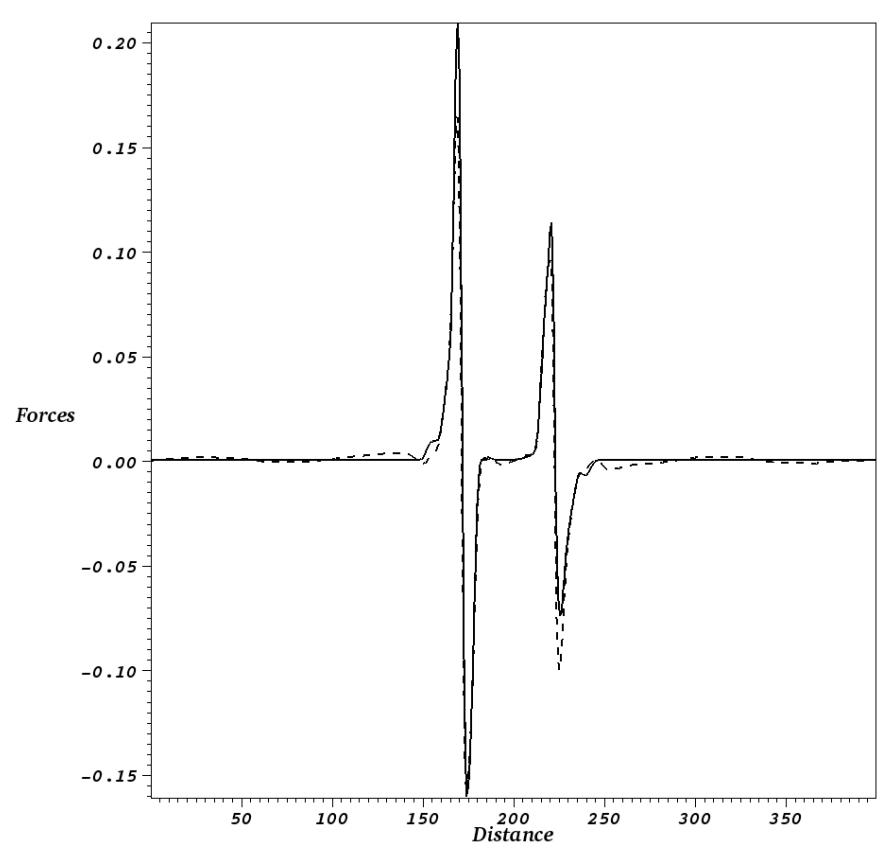

Fig. 5. The solid line shows $(\boldsymbol{j} \times \boldsymbol{B})_{x}$ and the dashed line, $\partial p / \partial x$. Both cuts are taken at the photosphere at time $t=338$ for experiment (iv).

separation has only a small effect on the steady-state drift velocity at the photosphere.

\subsubsection{Current densities}

In the original breakout model (Antiochos et al. 1999) described above, the initial condition is a potential quadrupolar field. In order to compare our work with others, we examine the 


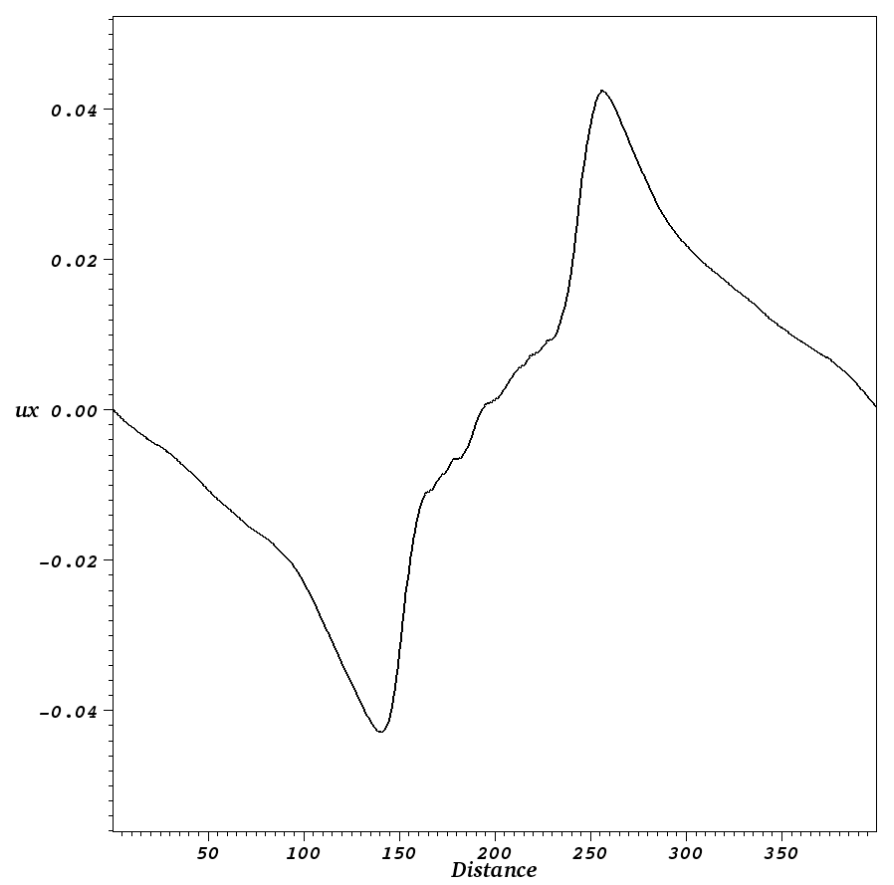

Fig. 6. The horizontal velocity at the photosphere at time $t=338$ for experiment (iv).

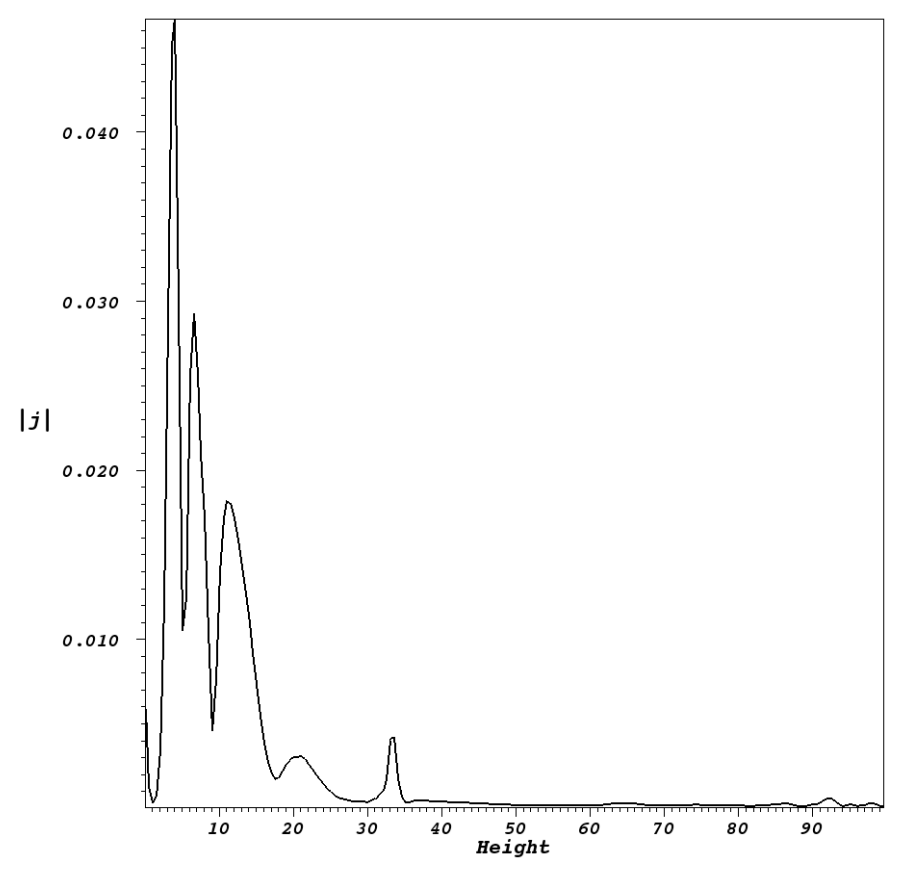

Fig. 7. The variation of current density with height for experiment (ii) at time $t=278$. The cut is taken through $x=200$.

magnitude of the current density and determine if the fields are close to potential.

Here we shall consider experiment (ii) with an initial axial separation of 40 . Figure 7 shows the variation of $|\boldsymbol{j}|$ with height, from the photosphere, through the centre of the simulation box, $x=200$. This also passes through the X-point. The currents below the corona $(y=0)$ are much stronger than those above it. Increasing in height from the photosphere, the current density decreases. This decrease is not monotonic, however, and is due to bands of flux that connect the two flanking tubes (see Fig. 2).

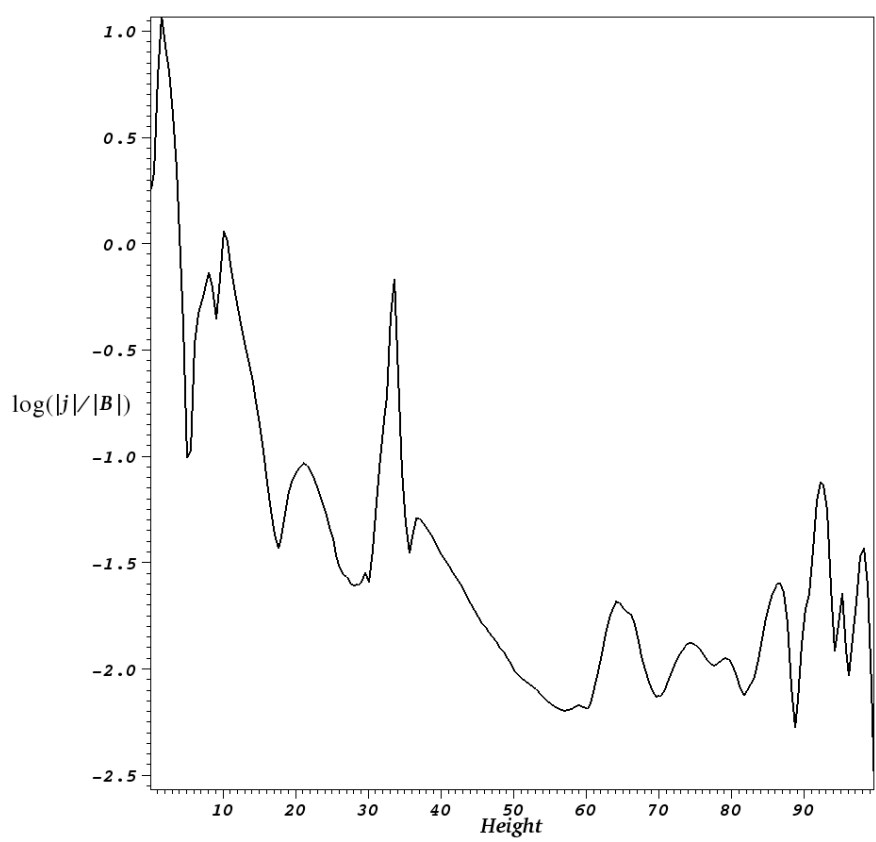

Fig. 8. The logarithmic variation of $j / B$ with height for experiment (ii) at time $t=278$. The cut is taken through $x=200$.

Above the corona, the current density continues to decrease until a sharp rise at a height of 33 . This is the position of the X-point. Beyond this the current becomes negligible compared with subcoronal values.

We will now look at how the magnitude of the current density compares with that of the magnetic field. Figure 8 shows the logarithmic variation of $|\boldsymbol{j}| /|\boldsymbol{B}|$ with height through the same cut as in Fig. 7. Below the corona there are bands of flux connecting the two flanking tubes. This results in peaks and troughs where the current dominates and is dominated, respectively, in the ratio with the magnetic field strength. Increasing in height towards the corona, there is a general decreasing trend in $|\boldsymbol{j}| /|\boldsymbol{B}|$. At a height of 33 there is a sharp increase corresponding to the X-point, where $|\boldsymbol{j}|$ increases and $|\boldsymbol{B}|$ decreases. Above the X-point, the curve decreases to -2 on the $\log _{10}$ scale and then fluctuates about -1.7 . The magnetic field strength becomes weaker with height but is still, from Fig. 8, nearly two orders of magnitude greater than the current density. Therefore, in the corona, with the exception of the X-point, the magnetic field is essentially potential. i.e. $\boldsymbol{j} \approx 0$. Below the corona, the field is not potential due to the presence of strong currents. As will be described in the following section, it is this region which contains vital physics that determines the final state of the system.

\subsection{Three tubes}

Now that the quadrupolar field has been created purely from flux emergence, the next step is to emerge a third flux tube up through the central arcade. The aim is to simulate the effect of shearing in the breakout model, without actually imposing any photospheric velocity shear. In the breakout model, although the central portion of the quadrupolar magnetic field is sheared, the arcades do not expand laterally at the base. This aids the reconnection neccessary for flux rope eruption because the increased magnetic pressure can only force the sheared region to expand into the $\mathrm{X}$-point, causing a current sheet to form there. In our flux emergence model, $\beta \sim O(1)$ at the photosphere. This means, as previously stated, that the plasma can move the field. When a third 


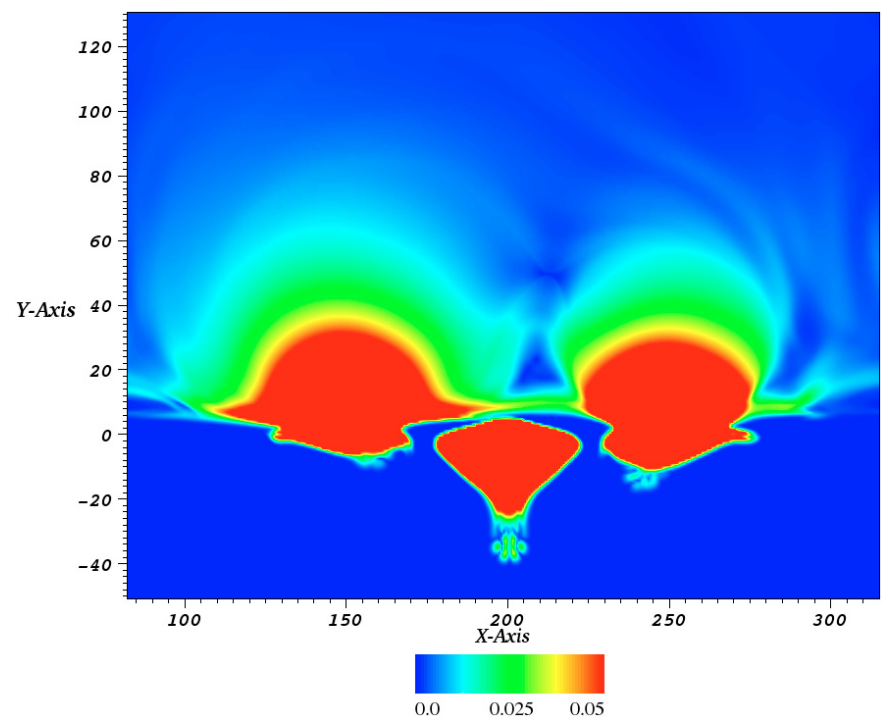

Fig. 9. The quadrupolar configuration with the middle tube at the photosphere at time $t=300$ for experiment (iv). The colourmap is of $|\boldsymbol{B}|$.

tube is inserted into the solar interior, below the central arcade of the quadrupole, it will rise and expand. The twist for the third tube is chosen so that it is in the opposite direction to that of the flanking tubes. This means that it won't reconnect with the side arcades upon emergence. When the third tube rises and emerges into the central arcade, it not only forces the field, already there, higher but it also interacts with the flanking tubes, causing them to move further apart. The scale of this movememt is different for the different cases. For experiments (i) and (ii), axial separations of 20 and 40, the middle tube will make contact with the flanking tubes below the photosphere and drive them further apart. For experiment (iii) with a separation of 60 , this interaction is less pronounced and in experiment (iv), with a separation of 80 , the middle tube can reach the photoshere without its field making direct contact with the flanking tubes. However, when the middle tube emerges through the photosphere, it expands both upwards and laterally. The emerged field does indeed push up into the $\mathrm{X}$-point and deform it into a current sheet. Reconnection occurs here and transports flux to the side arcades exactly in the same manner as in the breakout model. However, the emerged field also expands laterally and pushes the side arcades further apart. In the breakout model, the reconnected flux is added to the side arcades, increasing their pressure and so compressing the middle arcade. This does not happen in our simulation because the side arcades have already been moved further apart.

To study the effects of this sideways expansion, we shall now consider experiment (iv), where the interaction of the middle tube with the flanking tubes is expected to be the weakest out of the four experiments considered.

Figure 9 shows the quadrupolar configuration with the middle tube having reached the photosphere. Just above the middle tube there is a band of field connecting the two flanking tubes. This is due to the presence of strong currents in this region, which allows reconnection to occur. Above this region (and in the corona) the X-point can be seen just off-centre. Although the middle tube, for this case, has no direct contact with the flanking tubes, its expansion pushes the plasma away from it and increases the drift velocity of the flanking tubes. The velocity profile at the photosphere at time $t=300$ is given in Fig. 10. In this profile, the smaller peaks of the equilibrium flow can be seen.

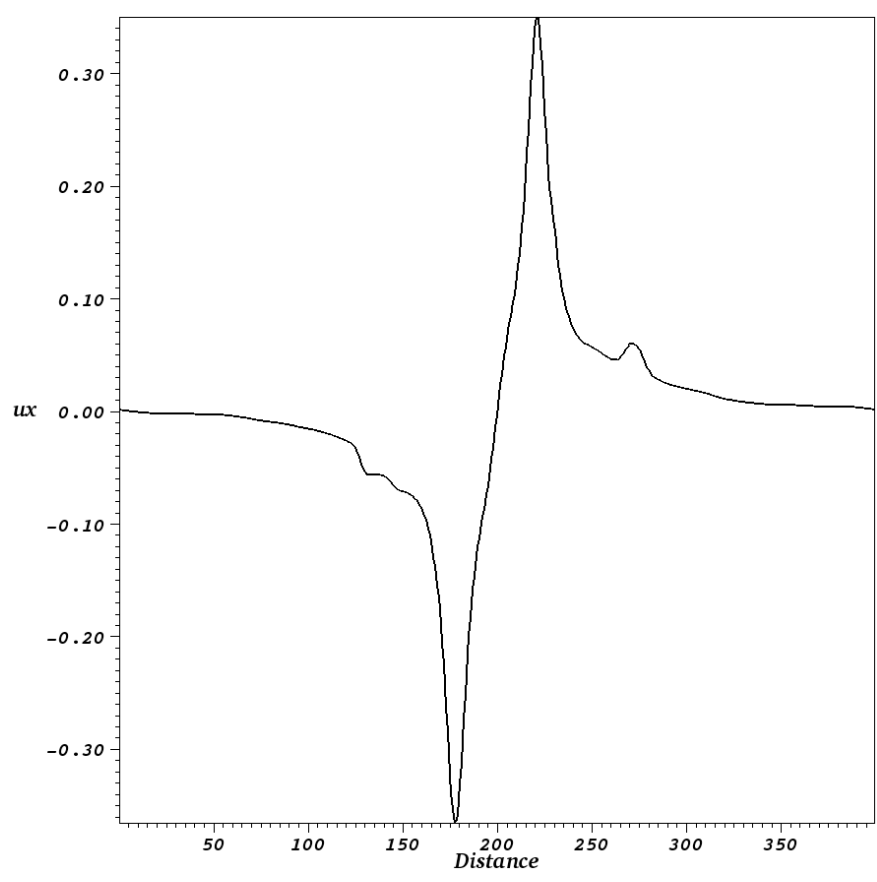

Fig. 10. The horizontal velocity profile at the photosphere at $t=300$.

We will now examine this experiment at a much later time when the middle tube has emerged and expanded. Figure 11 shows the configuration of the field at time $t=450$. It is clear that the middle tube has not only expanded upward but also laterally. By comparison with Fig. 9, the axes of the flanking tubes have each been pushed a distance of 20 further apart. The horizontal velocity at the photosphere is smaller in magnitude than it was at $t=300$ but it is still larger than the equilibrium velocity (see Fig. 6) produced by the quadrupole. The horizontal photospheric velocity at $t=450$ is displayed in Fig. 12. At the centre of this velocity profile there is a very small inflow. This, however, does not grow in time but flattens out. The horizontal photospheric velocity tends towards that for the equilibrium profile.

As the system expands laterally, the upward expansion has slowed down considerably. As the middle arcade expansion is driven by the emergence of a flux tube from a buoyancy instability, it will only expand for a limited time. Figure 13 displays the vertical velocities for $t=360$ and $t=450$ starting from the base of the photosphere $(y=0)$.

This combination of a lateral expansion of the arcades and a limited upward expansion means that the field compression required to drive reconnection and expel a flux rope does not happen. In other words, this self-consistent flux emergence model of magnetic breakout does not produce the eruption of a flux rope.

\section{Conclusions}

In this paper we have attempted to model magnetic breakout in $2.5 \mathrm{D}$ in a self-consistent manner. Standard models, such as those described in Sect. 1, have a quadrupolar field as their initial condition. This is dynamically altered by changing the boundary conditions to simulate shearing or flux emergence. Our model is based purely on multiple flux emergence, a common solar phenomenom, and the quadrupolar configuration is created as part of the process. When one tube emerges and expands, followed by an identical tube that emerges into the first's field, the resulting 


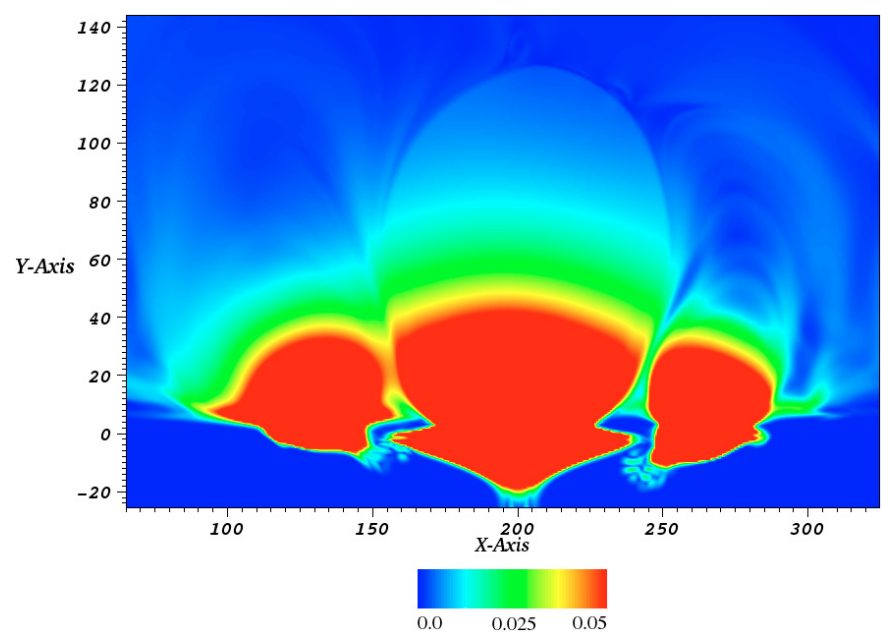

Fig. 11. The magnetic field of experiment (iv) at time $t=450$. The colourmap is of $|\boldsymbol{B}|$.

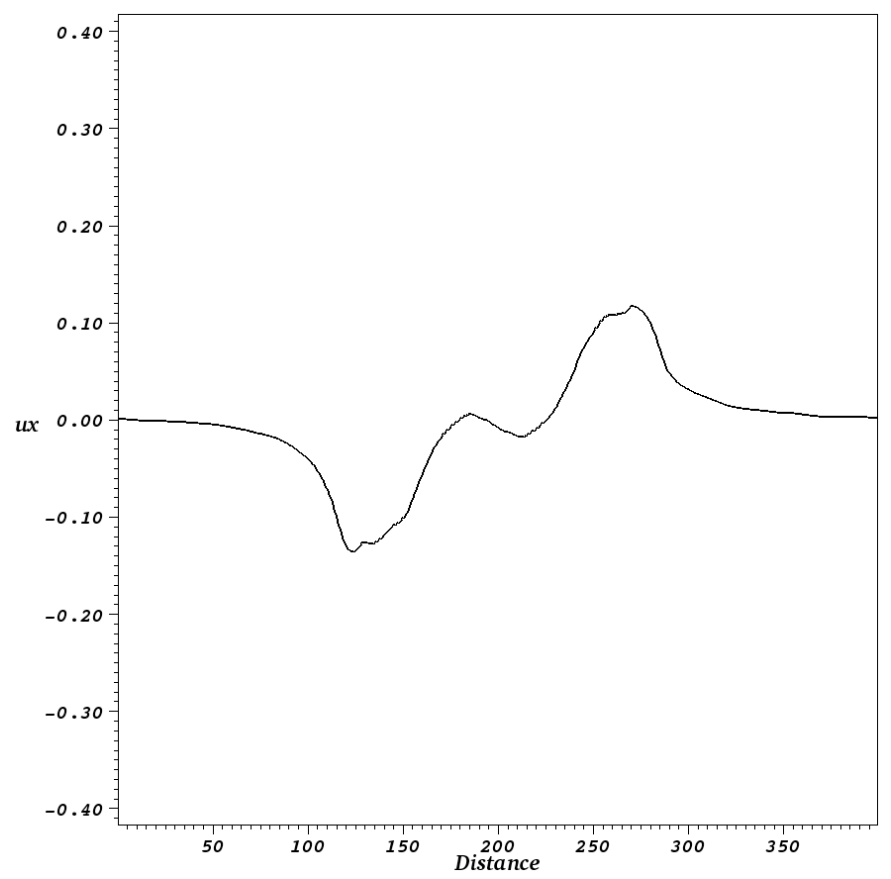

Fig. 12. The horizontal velocity profile at the photosphere at $t=450$.

equilibrium is a quadrupolar field. Experiments were performed to test the effect of the initial lateral separation of the two tubes. Each experiment results in a quadrupolar field where the system slowly expands. At the photosphere the sources gradually drift apart. The reason for this is that in our model, $\beta \sim O(1)$ at the photosphere. Therefore, if a flow is set up in the plasma, it can influence the magnetic field.

Rather than artificially impose shearing motions, we increase the magnetic pressure in the central arcade by emerging more flux here. Once the first two tubes have produced a quadrupolar equilibrium, a third tube of opposite twist is emerged below the central arcade of the quadrupole. Although other flux tube combinations are possible, we make this choice since it produces the correct magnetic configuration required by the breakout model. As this tube emerges, it pushes upwards to deform the X-point into a current sheet, as in the standard breakout scenario. The system, however, also expands laterally, pushing the

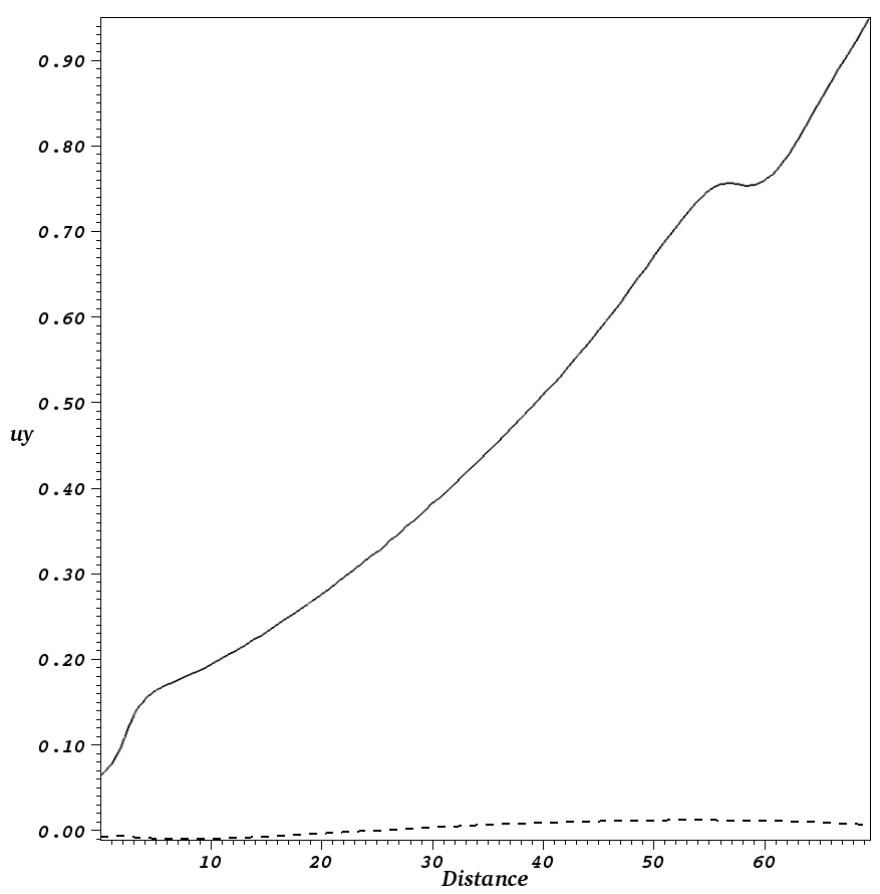

Fig. 13. The solid curve shows the vertical velocity through the central arcade at $t=360$. The dashed curve shows the same cut but at $t=450$.

quadrupolar arcades even further apart. The reason for this is, again, due to $\beta \sim O(1)$ at the photosphere and $\beta \gtrsim O(1)$ in the solar interior. This expansion acts against the central arcade pinching off to form a flux rope. This is in agreement with the results obtained by Zuccarello et al. (2008). They also do not attain the neccessary reconnection to pinch off a flux rope from the central arcade.

The upward expansion is also finite as the emergence is driven from a buoyancy instability. This in combination with the lateral expansion of the system means that this self-consistent flux emergence model does not produce breakout.

One philosophy in modelling the evolution of the magnetic field in the solar atmosphere is to impose boundary motions to trigger events such as CMEs. At the lower boundary, usually taken to be the photosphere, the magnetic field is fixed unless it is altered by artificially imposed motions. In the original breakout model, the side arcades remain fixed and constrain the central arcade until enough shearing has been applied to produce a flux rope. Our model differs from this approach by including the solar interior. This results in fields not being fixed at the photosphere. In the solar interior, $\beta \gtrsim O(1)$ and in the photosphere, $\beta \sim O(1)$. This means that flows in these regions can influence and move the magnetic field. As previously described, this is indeed what is found to occur in the numerical experiments. In our model, the footpoints at the photosphere of the side arcades diverge as the central arcade expands. This study has demonstrated that including the effects of the solar interior in models concerned with atmospheric events can be important and that the assumption of a field fixed at the lower boundary must be re-assessed.

Acknowledgements. D.M. acknowledges financial assistance from STFC and the Solaire Network. The computational work for this paper was carried out on the joint STFC and SFC (SRIF) funded cluster at the University of St Andrews (Scotland). 


\section{References}

Aly, J. J. 1991, ApJ, 375, L61

Antiochos, S. K., DeVore, C. R., \& Klimchuk, J. A. 1999, ApJ, 510, 485 Arber, T. D., Longbottom, A. W., Gerrard, C. L., \& Milne, A. M. 2001, J. Comput. Phys., 171, 151

Archontis, V., \& Török, T. 2008, A\&A, 492, L35

Archontis, V., Moreno-Insertis, F., Galsgaard, K., Hood, A., \& O'Shea, E. 2004, A\&A, 426, 1047

Archontis, V., Moreno-Insertis, F., Galsgaard, K., \& Hood, A. W. 2005, ApJ, 635,1299

Archontis, V., Hood, A. W., \& Brady, C. 2007, A\&A, 466, 367

Biskamp, D., \& Welter, H. 1989, Sol. Phys., 120, 49

Cook, G., Mackay, D. H., \& Nandi, D. 2009, ApJ, submitted

DeVore, C. R., \& Antiochos, S. K. 2008, ApJ, 680, 740

Fan, Y. 2001, ApJ, 554, L111

Forbes, T. G. 2000, J. Geophys. Res., 105, 23153

Galsgaard, K., Archontis, V., Moreno-Insertis, F., \& Hood, A. W. 2007, ApJ, 666,516

Li, Y., \& Luhmann, J. 2006, ApJ, 648, 732
Linker, J. A., Mikić, Z., Lionello, R., et al. 2003, Phys. Plasmas, 10, 1971 Lynch, B. J., Antiochos, S. K., DeVore, C. R., Luhmann, J. G., \& Zurbuchen, T. H. 2008, ApJ, 683, 1192

Mackay, D. H., \& van Ballegooijen, A. A. 2006a, ApJ, 641, 577

Mackay, D. H., \& van Ballegooijen, A. A. 2006b, ApJ, 642, 1193

MacNeice, P., Antiochos, S. K., Phillips, A., et al. 2004, ApJ, 614, 1028

Manchester, IV, W., Gombosi, T., DeZeeuw, D., \& Fan, Y. 2004, ApJ, 610, 588

Mikic, Z., Barnes, D. C., \& Schnack, D. D. 1988, ApJ, 328, 830

Murray, M. J., Hood, A. W., Moreno-Insertis, F., Galsgaard, K., \& Archontis, V. 2006, A\&A, 460, 909

Shibata, K., Tajima, T., Steinolfson, R. S., \& Matsumoto, R. 1989, ApJ, 345, 584 Sturrock, P. A. 1991, ApJ, 380, 655

Török, T., \& Kliem, B. 2003, A\&A, 406, 1043

Ugarte-Urra, I., Warren, H. P., \& Winebarger, A. R. 2007, ApJ, 662, 1293

van der Holst, B., Jacobs, C., \& Poedts, S. 2007, ApJ, 671, L77

Yeates, A. R., \& Mackay, D. H. 2009a, Sol. Phys., 254, 77

Yeates, A. R., \& Mackay, D. H. 2009b, ApJ, accepted

Zhang, Y., Zhang, M., \& Zhang, H. 2008, Sol. Phys., 250, 75

Zuccarello, F. P., Soenen, A., Poedts, S., Zuccarello, F., \& Jacobs, C. 2008, ApJ, 689, L157 